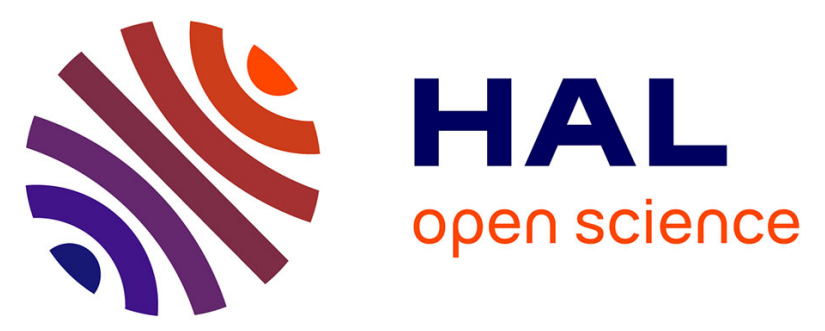

\title{
In vitro PUVA treatment triggers calreticulin exposition and HMGB1 release by dying T lymphocytes in GVHD: New insights in extracorporeal photopheresis
}

Céline Coppard, Dalil Hannani, Marion Humbert, Virginie Gauthier, Joël Plumas, Etienne Merlin, Françoise M. Gabert, Laurence Chaperot

\section{To cite this version:}

Céline Coppard, Dalil Hannani, Marion Humbert, Virginie Gauthier, Joël Plumas, et al.. In vitro PUVA treatment triggers calreticulin exposition and HMGB1 release by dying $\mathrm{T}$ lymphocytes in GVHD: New insights in extracorporeal photopheresis. Journal of Clinical Apheresis, 2019, 34 (4), pp.450-460. 10.1002/jca.21698 . hal-02361628

\section{HAL Id: hal-02361628 \\ https://hal.science/hal-02361628}

Submitted on 18 Dec 2020

HAL is a multi-disciplinary open access archive for the deposit and dissemination of scientific research documents, whether they are published or not. The documents may come from teaching and research institutions in France or abroad, or from public or private research centers.
L'archive ouverte pluridisciplinaire HAL, est destinée au dépôt et à la diffusion de documents scientifiques de niveau recherche, publiés ou non, émanant des établissements d'enseignement et de recherche français ou étrangers, des laboratoires publics ou privés. 


\section{Title Page}

\section{Title}

In vitro PUVA treatment triggers Calreticulin exposition and HMGB1 release by dying T lymphocytes in GvHD: new insights in Extracorporeal Photopheresis

\section{Authors}

Céline Coppard ${ }^{1,2}, \mathrm{PhD}$, Dalil Hannani ${ }^{1,2,3}, \mathrm{PhD}$, Marion Humbert ${ }^{1,2}$, Virginie Gauthier ${ }^{1,2}$, Joel Plumas $^{1,2,3}$, PhD, Etienne Merlin ${ }^{4}, \mathrm{MD}$, PhD, Françoise Gabert ${ }^{1,2}, \mathrm{PhD}$, Laurence Chaperot ${ }^{1,2}, \mathrm{PhD}$.

Affiliations:

1- Institute for Advanced Biosciences; Université Grenoble Alpes, INSERM U1209,CNRS UMR 5309, Grenoble-FRANCE

2- Etablissement Français du Sang Auvergne-Rhône-Alpes, Research and development, Grenoble-FRANCE

3- PDC*line Pharma, Grenoble-FRANCE

4- Centre Hospitalier Universitaire de Clermont-Ferrand, pôle Femme-Enfant, ClermontFerrand-FRANCE

\section{Corresponding author:}

Laurence Chaperot : Laurence.Chaperot@efs.sante.fr

Mailing address : Etablissement Français du Sang Auvergne-Rhône-Alpes, Research and development lab, 29 Av maquis du Grésivaudan , 38701 La Tronche, FRANCE

Tel : +33 (0)4 $76429416-$ Fax : +33 (0)4 76429449

\section{Institution at which the work was performed:}

Etablissement Français du Sang Auvergne-Rhône-Alpes, Research and development, GrenobleFRANCE

\section{Short Title}

Extracorporeal photopheresis-induced DAMPs

\section{Support:}

This work benefited from support of the French Government (ANRT) with CIFRE funding. This project was supported by Etablissement Français du Sang APR 2013 and Agence de Biomédecine grants. 


\section{Abstract}

Background: Extracorporeal photopheresis (ECP) is an effective therapy for Graft versus Host Disease (GVHD), based on infusion of UVA-irradiated and 8 methoxy-psoralen (PUVA)-treated leukocytes. Reinfusion of these apoptosing cells affects the functionality of pathogenic $T$ cells through poorly understood immunomodulatory mechanisms. Apoptosis is usually a silent, tolerance-associated process, but can also be immunogenic, depending on death-inducers and environmental context.

Methods: To understand ECP mechanisms of action, human alloreactive T cells generated in an in vitro model mimicking GVHD were used, as well as primary cells from GVHD patients. Cells were submitted to PUVA treatment and their phenotype and immunogenicity were analyzed, using cell culture and flow cytometry.

Results: In vitro PUVA treatment induced the expression of several Damage-Associated Molecular Patterns (DAMPs) by dying T cells (CRT, HMGB1, and to a lesser extent HSP70 and HSP90), especially upon $T$ cell activation, leading to their phagocytosis by macrophages and dendritic cells (DC). Allogeneic DCs pre-incubated with PUVA treated T cells induced comparable naive $T$ cell proliferation and polarization as control allogeneic DC.

Conclusion: Altogether, in our experimental settings, in vitro PUVA-treatment induces a partially immunogenic phenotype allowing phagocytosis of apoptotic cells by macrophages and DC, however not sufficient to induce dendritic cell maturation and $T$ cell activation. These data refine current models of ECP-mediated immune modulation and emphasize the need to further analyze PUVAtreated cell interactions with immune cells.

\section{Keywords:}

Extracorporeal photopheresis; Damage-Associated Molecular Patterns; apoptosis; Graft versus Host

Disease; dendritic cells 


\section{Abbreviations}

APC, antigen-presenting cell

CRT, calreticulin

CTCL , cutaneous T cell lymphoma

DAMPs, Damage-Associated Molecular Patterns

ECP , Extracorporeal photopheresis

ER endoplasmic reticulum

GVHD, Graft versus Host Disease

HMGB1, Human Recombinant | High-Mobility Group Box-1

HSP70 and HSP90, heat shock proteins 70 and 90

ivPUVA, in vitro treatment of cells with 8 methoxy-psoralen plus ultra violet $A$ irradiation

MLR, Mixed lymphocyte reactions

MoDC , monocyte-derived dendritic cell

PBMC, peripheral blood mononuclear cells

PDT, photodynamic therapy

PS, Phosphatidylserine

PUVA, 8 methoxy-psoralen plus ultra violet $A$ irradiation

SR, scavenger receptors

Treg, regulatory T cells 


\section{1-Introduction}

Extracorporeal photopheresis (ECP) is an autologous cell therapy in which leukocytes collected by cytapheresis are incubated with 8 methoxy-psoralen (8-MOP), irradiated with UVA, before reinfusion to the patient. ECP is used to treat diseases with circulating pathogenic T cells, such as cutaneous $T$ cell lymphoma (CTCL)(1), Graft-versus-Host Disease (GVHD) $(2,3)$, and various T-cell mediated disorders (organ transplant rejection (4), auto-immune (5) or inflammatory diseases (6)). Despite its widespread use, ECP mechanisms of action have long been a matter of debate (7). Indeed, ECP can be considered both as a immunizing or a tolerizing therapy, depending on the pathology considered, and recently, a new organization was funded focused on this unique cell therapy(8).

PUVA treatment (Psoralen - i.e. 8-MOP +UVA irradiation) induces T cell apoptosis. It has been shown that PUVA-treated T lymphocytes - including tumoral, allo-reactive or autoimmune T cells - undergo apoptosis within 24 to 48 hours, with an accelerated kinetics for activated cells (9). During ECP, only a fraction of total circulating leukocytes are treated, and thus only relatively few pathogenic $T$ cells are directly affected, indicating that direct elimination of the apoptotic pathogenic $T$ cells cannot be the sole mechanism responsible for therapeutic efficacy (10). This treatment nevertheless leads to potent and prolonged clinical responses with dampening of pathogenic $T$ cell activity, suggesting systemic ECP-induced immune-modulation, and highlights the central role of the presence of pathogenic T cells for treatment efficacy.

Several hypotheses have been put forward to explain ECP mechanisms of action: ECP is shown to be tolerogenic in the GVHD or auto-immunity context, while inducing anti-tumor responses in the CTCL context. Monitoring of regulatory T cells frequencies during ECP treatment has been performed in several trials. Some reports describe an increase in circulating Treg percentages over the course of several months during ECP treatment (11-14), predominantly in the context of GHVD; however, stable or decreasing Treg frequencies were reported in other pathologic contexts $(13,15)$. In most cases, Treg numbers display large inter-individual heterogeneity (16), and do not necessarily correlate with clinical responses (17). In the context of CTCL treatment, it has been suggested that ECP could induce a cytotoxic response directed towards the malignant clone (18). Whether immunizing or tolerizing, ECP should involve dendritic cells as central antigen-presenting cells(19). Using the Therakos device, Edelson et al. found that PUVA-treated monocytes differentiate into dendritic cells (MoDC), via interactions involving platelets, integrins, plasma proteins and the plastic vessel (20-22), these ECP-induced DC being efficient immunizing agent. In a recent study in murine model, it was shown that ECP was able to drive anti-cancer immune response, while using PUVAtreated tumor cells, in combination with untreated PBMC and platelets (23), mixed together, passed through a device named "transimmunization chamber" and cocultured overnight before intreavenous injection to tumor-bearing mice. In this model, treatment efficacy relies on extracorporeal device induced dendritic cells presenting Ag from PUVA-apoptotic malignant cells to prime CD4 and CD8 anti-tumor T lymphocytes. Besides these immunogenic ECP-induced DC, it was also shown that PUVA stimulates GILZ expression on MoDC, and down-regulation of CD80 and CD86 leading to immunosuppressive moDC function(24), and it could be interesting to consider modification of ECP apparatus to better control this polarization(19). In our settings, free of platelets, most PUVA-treated monocytes became apoptotic, with a delayed kinetics compared to $T$ cells, their apoptosis occurring 5 to 6 days following PUVA treatment (25). Moreover, the few surviving 
monocytes did not differentiate spontaneously into MoDC, while displaying no compromised functionality and in particular without acquiring immunosuppressive properties (25).

As a working model, it can be hypothesized that upon phagocytosis of pre-apoptotic PUVA-treated cells, the re-infused APC or the resident APC initiate a specific immunomodulatory program, targeting pathogenic $T$ cells without inducing generalized immunosuppression.

Apoptosis is usually a silent physiological process inducing tolerance (26). During apoptosis, dying cells express "eat-me" signals, allowing their recognition by phagocytes. Phosphatidylserine (PS), normally found on the inner leaflet of the plasma membrane, is exposed at the cell surface of apoptotic cells. PS is directly recognized by several receptors, such as stabilin 2, BAl1 and Tim-4, or indirectly by MerKT or $\alpha v \beta 5$ integrin (27). These receptors can cooperate to mediate an immunologically silent removal of dying cells by phagocytes, with secretion of anti-inflammatory cytokines, leading to regulatory $T$ cells differentiation/ expansion and active tolerance induction. Some of these PS receptors belong to the scavenger receptors (SR) family $(28,29)$, which are predominantly expressed by myeloid cells. They mediate endocytosis, phagocytosis, adhesion, or signaling leading to the elimination of non-self or altered-self targets. In addition to their patternrecognition and phagocytic functions, SR also regulate inflammatory signaling. On the other hand, it has become evident that apoptosis can also be immunogenic, depending on death inducers and environmental context (30). For example, anthracyclines, oxaliplatin or gamma-radiation induce the surface expression or secretion of DAMPs (damage-associated molecular patterns), such as calreticulin (CRT), heat shock proteins (HSP), high mobility group box 1 (HMGB1), or ATP. Calreticulin, a chaperone found in the endoplasmic reticulum (ER), rapidly translocates to the plasma membrane following ER stress induced during some forms of apoptosis $(31,32)$. Early exposure of CRT is a hallmark of Immunogenic Cell Death (ICD). CRT is recognized by CD91 expressed by phagocytes, allowing apoptotic cell phagocytosis. ATP released during apoptosis is recognized by purinergic receptors, activating the NLRP3 inflammasome and stimulating the release of IL-1 $\beta$ and IL-18 by phagocytes (33). The nuclear protein HMGB1 released extracellularly during late apoptosis or necrosis acts as an alarmin, mediating phagocyte chemo-attraction and activation upon its recognition by TLR2, TLR4 and RAGE.

In order to better characterize ECP mechanisms of action, we set up an in vitro model of alloreactive $T$ cells in mixed lymphocyte reactions to mimic alloreactive T cells in GVHD, and analyzed whether in vitro PUVA (ivPUVA) treatment could induce their expression or secretion of ICD signals. We also studied the ability of ivPUVA-treated alloreactive T cells to trigger DC maturation and analyzed the subsequent DC-induced T cell polarization. 


\section{2-Materials and methods}

Medium and Reagents

Cultures were performed in RPMI 1640 Glutamax supplemented with $1 \mathrm{mmol} / \mathrm{L}$ sodium pyruvate, nonessential amino acids, $20 \mathrm{ng} / \mathrm{mL}$ gentamycin, $50 \mu \mathrm{M}$ beta-mercapto-ethanol, and $10 \%$ heat inactivated fetal calf serum (referred to as complete medium).The 8-Methoxy-psoralen used for ivPUVA treatment was from Sigma-Aldrich.

Immunophenotyping was performed by using antibodies directed towards: CD3, HLA-DR (BD Biosciences), calreticulin, HSP-90 and HSP-70 (AbCam). CD209, CD14, CD40, CD80 and the FITCAnnexinV and 7AAD kit were purchased from Beckman Coulter.

Flow cytometric experiments and analyses were performed using an 8-color FACSCanto II flow cytometer with the Diva or the Flow Jo software (BD Biosciences).

\section{Cells, mixed lymphocytes reactions and antigen-presenting cell (APC) generation}

Blood samples were collected from adult healthy volunteers, and patients with extensive chronic GVHD who gave their informed consent in accordance with the Declaration of Helsinki (cell collections declared to the French Health ministry (GRE-DC-2008-787, NCT 00824954, CPP Sud Est I : ref CPP JV-TV/2008-273 authorization). Cord blood not suitable for cord blood banking was obtained from Bourgogne Franche-Comte EFS (GRE-DC-2011-1487). PBMC were isolated by density gradient on lymphocyte separation medium, and cryopreserved until use, by standard freezing methods (37.5\% FCS, 10\% Dimethyl sulfoxide). T cells and monocytes enrichment cocktails EasySep kit (Stem Cell Technologies Inc) were used for cell separation.

Mixed lymphocyte reaction (MLR), a simple and efficient in vitro model for the study of T-cell activation and proliferation, was used to obtain alloreactive-activated T lymphocytes. PBMC from healthy donors were co-cultured during 6 days with irradiated ( $\gamma$-irradiation, 30Gy) allogeneic PBMC (1:1 ratio), 96-round bottomed plates. Dead cells were removed by density gradient on lymphocyte separation medium. CD25 was expressed by less than $10 \%$ of T cells purified from PBMC, whereas it was expressed by more than $50 \%$ of the T cells after MLR (data not shown). Therefore we considered purified T cells extracted from fresh PBMC as 'resting T cell', whereas T cells obtained after MLR were considered as 'alloreactive activated T cells'. Activated T cells were purified after the MLR with EasySep kit (Stem Cell Technologies Inc).

Monocyte-derived dendritic cells (MoDC) were generated by culturing monocytes in complete medium supplemented with $10 \%$ FCS in presence of GM-CSF (500 U/mL, Miltenyi) and IL-4 (10 $\mathrm{ng} / \mathrm{mL}$, Miltenyi) for 6 days. IL-4 was added at day 2 and 5 of culture, and MoDC were harvested at day 7. Macrophages were generated by a 7-day culture of monocytes with GM-CSF $(500 \mathrm{U} / \mathrm{mL})$. These cultures were performed in low attachment $25 \mathrm{~cm}^{2}$ flasks (Corning).

\section{$\underline{\text { In vitro PUVA (ivPUVA) Treatment }}$}

PBMC or purified T cells were seeded at $1.10^{6} \mathrm{cells} / \mathrm{mL}$ in complete medium, in 24-well culture plates. Cells were incubated for $15 \mathrm{~min}$ at $37^{\circ} \mathrm{C}$ with $200 \mathrm{ng} / \mathrm{mL}$ of $8-\mathrm{MOP}$ and exposed to $2 \mathrm{~J} / \mathrm{cm}^{2} 365-\mathrm{nm}$ UVA radiation (Bio Sun, Vilbert-Lourmat), as previously described $(9,25)$. After ivPUVA treatment, cells were washed and cultured in complete medium at $37^{\circ} \mathrm{C}$ in $5 \% \mathrm{CO} 2$ atmosphere. 


\section{Calreticulin and HSP expression}

Flow cytometry was used to measure surface expression of calreticulin, HSP-70 and HSP-90. Cells were gated on $\mathrm{CD}^{+}$7-AAD negative cells to focus on $\mathrm{T}$ lymphocytes that retained their membrane integrity.

\section{$\underline{\text { HMGB1, ATP and cytokine secretion assays }}$}

Culture supernatants were harvested and stored at $-20^{\circ} \mathrm{C}$. HMGB1 was measured by ELISA (IBL international). ATP content was measured with Bioluminescent somatic cell assay kit (Sigma-Aldrich). Cytokines (IL-6, IL-8, IL-10, TNF $\alpha$, IL-12p70, IL-1 $\beta$, IL-4, IL-17, or IFN $\gamma$ ) were quantified in culture supernatants by cytometric bead array (CBA, BD Biosciences).

\section{Phagocytosis and MoDC maturation assay}

For phagocytosis, APC (macrophages or MoDC) were incubated for 1 to 4 hours at $37^{\circ} \mathrm{C}$ or $4^{\circ} \mathrm{C}$ with CFSE (Life technologies)-labeled purified activated or resting T lymphocytes (ratio 1:1, culture tubes, Falcon), treated or not by ivPUVA. Phagocytosis was stopped (washing at $4^{\circ} \mathrm{C}$ in PBS), APC were labeled (MoDC with CD209, and macrophages with CD14), T cells with BV421-CD3. After washing, CFSE fluorescence was analyzed on gated APC. CD3-positive APC were excluded from the analysis as they had likely formed conjugates with $T$ cells but had not necessarily internalized cellular material, as previously described by Lui et al.(34).

MoDC maturation was measured after $24 \mathrm{~h}$ and $48 \mathrm{~h}$ of coculture with purified activated or resting $\mathrm{T}$ lymphocytes (ratio 1:1), treated or not by ivPUVA. Poly I:C ( $25 \mu \mathrm{g} / \mathrm{ml}$, Invivogen) was used as positive control. Cells were harvested, and stained with CD209, CD40 and CD80 antibodies. Mean fluorescence intensity of CD40 and CD80 was quantified on gated CD209+ cells, and expressed as fold increase in comparison with MoDC incubated in medium alone.

\section{$\underline{\text { Naive T cell proliferation and polarization }}$}

Purified activated or resting T lymphocytes were treated or not by ivPUVA, and cultured for $24 \mathrm{~h}$ to allow DAMPs expression and release. Autologous MoDC were then added $(5,000$ cells of each subset in $100 \mu \mathrm{L}, 96-$ well round bottomed plates) for $24 \mathrm{~h}$ to allow MoDC maturation. Purified allogeneic CD4+ naive $T$ cells from cord blood were then added to these co-cultures $(50,000$ naive $T$ cell/well, APC: naive T cell ratio $=1: 10$, final culture volume $: 200 \mu \mathrm{L}$ ). . Proliferation was measured after 6 days by ${ }^{3} \mathrm{H}$-Thymidine incorporation (addition of $1 \mu \mathrm{Ci}{ }^{3} \mathrm{H}$-Thymidine (PerkinElmer) during the last 18 hours of culture). In another setting, cells were stimulated at day 6 for 5 hours with PMA $(5 \mathrm{ng} / \mathrm{mL})$ and ionomycin $(5 \mu \mathrm{g} / \mathrm{mL}$ ) (both from Sigma Aldrich), and supernatants were harvested to measure their cytokine contents.

\section{$\underline{\text { Statistical analysis }}$}

Data are presented in percentages, concentrations or fold changes, with mean and standard deviation shown. All statistical analyses were performed with the GraphPad prism software. Mann and Whitney $U$ test, or ANOVA analysis was used, followed by Tukey multiple comparison tests to compare the different groups. Significance level was set at $p<0.05(*), p<0.01\left({ }^{* *}\right)$ or $p<0.001(* * *)$. 


\section{3-Results}

\section{1 ivPUVA induces apoptosis in resting and allo-reactive activated T cells}

In order to partially recapitulate the GVHD situation, mixed lymphocyte reactions (MLR) were used to generate activated alloreactive T cells in vitro. Apoptosis was measured on CD3+ T lymphocytes, 24 and 48 hours after ivPUVA treatment of resting or activated cells. Following ivPUVA treatment, resting and activated T cells exposed PS (Annexin-V labeling), and lost membrane integrity (7-AAD incorporation), demonstrating the induction of apoptotic cell death (Figure 1A). After a 24hincubation, $29 \%$ and $71 \%$ of resting and activated T cells died (Annexin-V positive, and/or 7AAD positive). These percentages increased at $48 \mathrm{~h}$, reaching 46 and $90 \%$, respectively (Figure 1B). The death kinetics was more rapid, and the percentage of apoptosis was statistically higher (Annova test, $p<0.01$, Tuckey post-test) for ivPUVA-treated activated alloreactive $T$ cells (i.e. 'pathogenic' $T$ cells) compared to ivPUVA-treated resting T cells, recapitulating the T cell behavior observed in GVHD patients (9), and validating MLR-activated alloreactive T cells as an in vitro model of GVHD.

\section{2 ivPUVA induces DAMPs expression in human alloreactive and tumoral T cells}

To explore the immunogenicity of apoptotic ivPUVA-treated cells, DAMPs expression and secretion were analyzed. Resting and alloreactive activated cells were treated by ivPUVA, and the expression of calreticulin was measured by flow cytometry 24 and 48 hours post-treatment (Figure 2A). Calreticulin exposure was analyzed only on T cells which retained their membrane integrity (7-AAD negative cells). Indeed, most 7AAD+ cells appeared CRT positive, but in this case it is not possible to differentiate external or intracellular expression (not shown). In resting 7AAD-negative T cells, 9\% of cells expressed calreticulin $24 \mathrm{~h}$ following ivPUVA treatment, and $15 \%$ after $48 \mathrm{~h}$. A significantly higher percentage of calreticulin expression was measured on activated T cells following ivPUVA treatment: $14 \%$ and $27 \%$ of calreticulin expressing cells were detected at 24 and 48 hours, respectively (Figure 2B, $p<0.01$, Mann and Whitney $U$ test). Of note, CRT was not detected on T cells 5 hours after ivPUVA treatment (not shown).

As comparison, ivPUVA-treated samples from two GVHD patients were analyzed, and HLA-DR expression was used to identify activated (alloreactive) T cells (Figure 3A, B). A slightly higher percentage of calreticulin-expressing $T$ cells was detected on activated ( 45 and $59 \%$ ) compared to resting cells (31 and 47\%). In another pathological context, we found that ivPUVA-treated T cells from 3 CTCL patients also up-regulated calreticulin (9 to 37\%) 24 hours after ivPUVA treatment (Supplementary figure $1 \mathrm{~A}, \mathrm{~B}$ ).

HMGB1 was measured in the supernatant of resting or alloreactive activated cells following ivPUVA treatment. HMGB1 was detected in resting cell supernatants after $24 \mathrm{~h}$ (mean: $26 \mathrm{ng} / \mathrm{ml}$ ), and 48 hours $(29 \mathrm{ng} / \mathrm{ml}$ ) post-treatment (Figure $2 \mathrm{C}$ ). The release of HMGB1 following ivPUVA-treatment was higher in the supernatant of alloreactive-activated cells $(35$ and $54 \mathrm{ng} / \mathrm{ml}$, after 24 and 48 hours, respectively). Both HSP-70 and HSP-90 were induced on activated T cells 48 hours following ivPUVAtreatment (as measured by flow cytometry analysis of surface expression- supplementary Figure 2), but ATP (measured by a bioluminescence assay and HPLC), was undetectable in all samples (data not shown).

Altogether, ivPUVA-treatment induces DAMPs expression (CRT and HSP70 and 90) or release (HMGB1) by T cells in different contexts, and at higher levels when treated T cells are activated (i.e. pathogenic alloreactive T cells). 
3.3 Antigen-presenting cells phagocytize ivPUVA-treated, dying cells and partially mature The capacity of APC (macrophages and DC differentiated from monocytes) to phagocytize resting and activated ivPUVA-treated cells was evaluated by flow cytometry (Figure 4A, B). Phagocytosis was measured from 30 min to 4 hours, and determined by the percentage of CFSE positive cells ( $T$ cells were stained by CFSE prior to the assay) among CD3-negative CD14- or CD209-positive APC (to exclude APC and T cell conjugates). After 4 hours, $20 \%$ and $42 \%$ of the macrophages had phagocytized resting $T$ cells and activated $T$ cells, respectively (Figure $4 C$ ), indicating that macrophages engulfed alloreactive-activated ivPUVA-treated $T$ cells more efficiently and more rapidly than resting $T$ cells. A similar result was obtained when using MoDC (Figure 4D), with $12 \%$ of ivPUVA-treated resting T cell phagocytosis observed after 4 hours, compared to $39 \%$ for alloreactive activated T cells. Microscopic examination of the APC showed that whole T cells were engulfed, ruling out transfer of microvesicles or trogocytosis (not depicted). The more efficient phagocytosis of activated compared to resting T cells may be related to the observed differential CRT exposure by these ivPUVA-treated T cells.

The capacity of ivPUVA-treated cells to induce MoDC maturation was evaluated by measuring CD40 and CD80 on MoDC following 48-hour co-cultures. Resting T cells, treated or not by ivPUVA, did not induce MoDC maturation. Conversely, activated T cells induced an up-regulation of CD40 and CD80 expression on MoDC (2.5 and 1.5 fold increase, respectively), however ivPUVA-treated, activated T cells did not trigger DC maturation. Furthermore, the secretion of inflammatory cytokines (IL-12, IL-6, TNF- $\alpha$ ) was not altered compared to DC alone, and IL-10 secretion induced by incubation with activated T cells was abrogated when the T cells were ivPUVA-treated (supplementary Figure 3 ).

3.4 ivPUVA-induced dying cells do not modulate naive T cells proliferation or polarization The ability of MoDC to stimulate and polarize naive T cells from allogeneic cord blood was measured after pre-incubation with autologous ivPUVA-treated resting or activated T cells (ratio 1:1, 24h preincubation) (Figure 5A). MoDC alone efficiently induced the proliferation of naive $T$ cells (mean: $56,782 \mathrm{cpm}$ ). When MoDC were pre-incubated with resting T cells, whether treated or not by ivPUVA, proliferation of allogeneic naive $T$ cell was similar to the proliferation observed in the control condition (medium = MoDC alone i.e. not pre-incubated with any $T$ cells). In line with the upregulation of costimulatory molecules on MoDC induced by activated T cells, a higher proliferation of naive T cells was induced in this condition $(85,023 \mathrm{cpm})$; however, MoDC pre-incubated with ivPUVAtreated, activated $\mathrm{T}$ cells induced a comparable $\mathrm{T}$ cell proliferation as in the control condition (Figure $5 B)$.

Naive T cell polarization was measured upon co-culture with MoDC pre-incubated with resting or activated ivPUVA-treated, or non-treated T cells. Increased levels of TNF $\alpha$, IFN $\gamma$ and IL-4 were detected in the supernatants of co-cultures with MoDC and untreated resting or activated T cells. However, no such secretion was observed when T cells were treated by ivPUVA. Similar levels of IL10 were detected in all co-cultures, albeit heterogeneously (Figure 5C). 


\section{4-Discussion}

Photopheresis, a safe and efficient therapy initially used to treat CTCL, has been approved for several T cell-mediated diseases, including GVHD, solid organ transplant rejection and some auto-immune disorders. However the precise mechanisms of action of ECP remain elusive, hampering its wider implementation. ECP is particularly interesting in that it is not a global immunosuppressive therapy, preserving patients' general immunocompetence (35). This study aimed at dissecting the interaction between ECP-treated T cells and APC, and in particular it evaluated whether immunogenic features of cell death are induced upon ivPUVA treatment, potentially leading to a specific immunomodulatory response directed toward pathogenic $T$ cells (36). Apoptotic cell death accompanied by Calreticulin exposure, HMGB1 release and ATP secretion has been described as immunogenic, eliciting dendritic cell maturation and Th1 polarized adaptive immune responses. Such phenomenon has been mostly described in murine models, where cancer cell lines treated by doxorubicin, mitoxantrone or photodynamic therapy (PDT) were shown to be immunogenic.

We used mixed lymphocyte reactions to generate alloreactive T lymphocytes, as in these cultures thousands of alloreactive CD4+ and CD8+ T cell clones are amplified (37), generating large quantities of alloreactive T cells. In line with our hypothesis, we found that ivPUVA-treated T cells exposed CRT at their surface. CRT exposure was detected at the surface of early apoptotic cells following ivPUVA treatment in several models (primary T cells, alloreactive activated T cells from GVHD patients or in vitro-generated alloreactive T cells, and CTCL cells). CRT expression by human cells in the context of ICD has been reported on a melanoma cell line following infection with oncolytic vaccinia viruses (38), and on a bladder carcinoma cell line following hypericin-based photodynamic therapy $(39,40)$. CRT exposure at the surface of human acute myeloid leukemia blasts has also been correlated to enhanced autologous $T$ cell responses (41), independently of chemotherapy treatment. Detection of CRT at the surface of various kinds of ivPUVA-treated T-cells is very interesting and intriguing, and to our knowledge it is the first evidence of CRT expression on pre-apoptotic non-malignant human primary cells. Upon ivPUVA treatment, calreticulin exposure was accompanied by HMGB1 release, and both DAMPs were detected at higher levels with alloreactive-activated T cells than with resting $T$ cells. It is worth noting that in our in vitro allogeneic model non-treated activated T cells expressed higher levels of CRT than resting T cells, as well as for HLA-DR positive cells from GVHD patients, thus confirming the expression of CRT at the surface of activated T cells (42). In our hands, the supernatant of ivPUVA-treated cells did not contain ATP. This cannot be explained by ATP degradation by ectonucleotidases such as CD73 or CD39 (43) since we did not detect any ADP or AMP in ivPUVA-treated cells supernatants (not shown). In hypericin-based PDT, CRT exposure and HMGB1 release were accompanied with ATP secretion by dying cells (40); on the contrary, ATP secretion was not detected in the oncolytic virus-induced cell death model (38), despite dendritic cell maturation being induced in both instances.

Since ivPUVA-treatment of T cells induced CRT expression at the surface of dying cells, we asked whether APC could phagocytize ivPUVA-treated T cells. PUVA-treated cells re-infused to the patient first go into the lungs, before relocating to the spleen and liver (44), hence we focused our analysis on phagocytosis by the tissue-resident macrophages and DC, and used in vitro culture of monocytes with GM-CSF or GM-CSF+IL4 as in vitro model for these APC. For the first time, we provide evidence of ivPUVA-treated cell phagocytosis by macrophages and DC. Both phagocytes engulf ECP-induced apoptotic cells with a similar kinetics and efficacy. Interestingly, following ivPUVA-treatment, phagocytosis of activated compared to resting T cells was higher, in correlation with their higher level 
of CRT expression. Phagocytosis was highly efficient, and such efficient phagocytosis by DC has also been described for hypericin-PDT treated murine cells, and was mediated partially by CRT exposure (39).

In our model, despite expression of several DAMPs by dying cells, DC co-cultured with ivPUVAtreated cells did not mature. This result is in agreement with the observations published by Lamioni et al, who found that DC in contact with ECP-treated PBMC from patients did not up-regulate costimulatory molecule expression, but rather up-regulated HLA-DR molecules and displayed a tolerogenic phenotype (45). While remaining in an immature or semi-mature state, DC co-cultured with ivPUVA-treated cells did not secrete significantly different levels of inflammatory or antiinflammatory cytokines compared to immature DC. The absence of IL-1 $\beta$ secretion (not shown) is in line with the lack of ATP release by dying cells, as in the context of ICD extracellular ATP is a key factor promoting the release of IL-1 $\beta$ and IL-18 by APC (46). It is noteworthy that DC in contact with live activated T cells up-regulate CD40 and CD86, a phenomenon that could be related to DC licensing by, among others, members of the TNF family and IFN $\gamma$ secretion by T cells (47). Strikingly, such up-regulation did not occur anymore when activated $T$ cells were treated by ivPUVA, and despite HMGB1 release, these cells did not induce DC maturation.

In line with the absence of DC maturation induced by contact with ivPUVA-treated cells, there was no difference in the proliferation of naive T lymphocytes in allogeneic MLR induced by immature DC, and DC pre-incubated with ivPUVA-treated T lymphocytes, whether activated or not. Neither was there a difference in T cell polarization (Th1, Th2 or Treg) when measured at the MLR endpoint. Here again, these data indicate that apoptosis induced by ivPUVA treatment impaired the alloreactive activated $T$ cell ability to prime DC for $T$ cell activation. One limit of our study may be the use of monocyte-derived dendritic cells. Indeed, DCs comprise several subsets, with different roles in the presentation of antigens derived from pathogens, vaccines, and self-tissues. Monocytes are able to differentiate into DC when properly stimulated in vitro, and monocyte-derived-dendritic cells obtained after culture with GM-CSF and IL-4 are often used as surrogate for dendritic cells since they can be easily generated in large numbers(48). They may represent the equivalent of the inflammatory dendritic cells identified in mice(49), and may present different functionality compared to conventional myeloid or plasmacytoid dendritic cells. These GM-CSF+IL-4 moDC could be different from the platelet-induced dendritic cells derived from monocytes, as observed in the ECP Transimmune device (22). The in vitro model we chose here recapitulates the main steps of the process, but may not reflect all aspects of ECP as performed in the clinics.

It may be interesting to evaluate in an in vivo setting where and when re-injected PUVA-treated T cells encounter APC, as only a narrow window of time may exist during which PUVA-activated pathogenic $T$ cells can cross-talk with APC and induce their maturation, while at the same time expressing eat-me signals allowing their capture by these APC (36). In the experimental transimmunization device developed by Edelson et al., ECP efficacy relies on extracorporealdifferentiated DC that are incubated overnight with PUVA-treated malignant cells, allowing the capture, processing and presentation of tumor-Ag to $T$ cells, a device that seems attractive for improving ECP process. In our in vitro setting, the observed partial release of DAMPs by ivPUVAtreated cells fails to induce DC maturation; however, during ECP procedure as it is performed in clinics, it would be very interesting to decipher which are the APC involved in T cell priming, since 
they could originate from ECP-treated monocytes, or be the non-treated resident APC rendered immunogenic by the inflammatory milieu (due to systemic disease-associated immune activation).

In conclusion, we show here that ivPUVA-treatment of T lymphocytes induces the expression of CRT and the secretion of HMGB1 by dying cells, but no secretion of ATP. This unique apoptotic phenotype promotes very efficient phagocytosis of apoptotic cells by macrophages and DC but is not followed by DC maturation and T cell activation in our experimental settings. Further in vivo evaluations are still necessary to decipher the precise mechanisms of action of ECP, preferably in an inflammatory context recapitulating the clinical situation.

\section{Acknowledgements}

The authors would like to thank all healthy volunteers and patients for participation in the study, and staff in EFS in Grenoble, Valence, St Ismier and Besançon, who helped our research. We also thank Olivier Manches for correcting our manuscript. This work benefited from support of the French Government (ANRT) with CIFRE funding. This project was supported by Etablissement Français du Sang APR 2013 and Agence de Biomédecine grants. 


\section{References}

1. Alfred A, Taylor PC, Dignan F, et al. The role of extracorporeal photopheresis in the management of cutaneous t-cell lymphoma, graft-versus-host disease and organ transplant rejection: A consensus statement update from the uk photopheresis society. British journal of haematology. 2017; 177:287-310.

2. Kitko CL, Levine JE. Extracorporeal photopheresis in prevention and treatment of acute gvhd. Transfusion and apheresis science : official journal of the World Apheresis Association : official journal of the European Society for Haemapheresis. 2015;52:151-156.

3. Radojcic V, Pletneva MA, Couriel DR. The role of extracorporeal photopheresis in chronic graft-versus-host disease. Transfusion and apheresis science : official journal of the World Apheresis Association : official journal of the European Society for Haemapheresis. 2015;52:157-161.

4. Patel J, Klapper E, Shafi $\mathrm{H}$, et al. Extracorporeal photopheresis in heart transplant rejection. Transfusion and apheresis science : official journal of the World Apheresis Association : official journal of the European Society for Haemapheresis. 2015;52:167-170.

5. Adamski J, Kinard T, Ipe T, et al. Extracorporeal photopheresis for the treatment of autoimmune diseases. Transfusion and apheresis science : official journal of the World Apheresis Association : official journal of the European Society for Haemapheresis. 2015;52:171-182.

6. Fowler S, Jones J, Hull PR, et al. Extracorporeal photopheresis for the treatment of crohn's disease. Transfusion and apheresis science : official journal of the World Apheresis Association : official journal of the European Society for Haemapheresis. 2015;52:183-186.

7. Heshmati F. Updating ecp action mechanisms. Transfusion and apheresis science : official journal of the World Apheresis Association : official journal of the European Society for Haemapheresis. 2014;50:330-339.

8. Raval JS, Ratcliffe NR. Extracorporeal photopheresis and personalized medicine in the 21st century: The future's so bright! Journal of clinical apheresis. 2018;33:461-463.

9. Hannani D, Merlin E, Gabert F, et al. Photochemotherapy induces a faster apoptosis of alloreactive activated $t$ cells than of nonalloreactive resting $t$ cells in graft versus host disease. Transplantation. 2010;90:1232-1238.

10. Marshall SR. Technology insight: Ecp for the treatment of gvhd--can we offer selective immune control without generalized immunosuppression? Nature clinical practice Oncology. 2006;3:302-314.

11. Biagi E, Di Biaso I, Leoni V, et al. Extracorporeal photochemotherapy is accompanied by increasing levels of circulating $c d 4+c d 25+$ gitr+foxp $3+c d 621+$ functional regulatory t-cells in patients with graft-versus-host disease. Transplantation. 2007;84:31-39.

12. Rao V, Saunes M, Jorstad S, et al. Cutaneous $t$ cell lymphoma and graft-versus-host disease: A comparison of in vivo effects of extracorporeal photochemotherapy on foxp3+ regulatory t cells. Clin Immunol. 2009;133:303-313.

13. Quaglino $P$, Comessatti A, Ponti R, et al. Reciprocal modulation of circulating $\mathrm{cd} 4+\mathrm{cd} 25+$ bright $t$ cells induced by extracorporeal photochemotherapy in cutaneous $\mathrm{t}$-cell lymphoma and chronic graft-versus-host-disease patients. International journal of immunopathology and pharmacology. 2009;22:353-362.

14. Schmitt S, Johnson TS, Karakhanova S, et al. Extracorporeal photophoresis augments function of $c d 4+c d 25+f o x p 3+$ regulatory t cells by triggering adenosine production. Transplantation. 2009;88:411-416.

15. Dieterlen MT, Garbade J, Misfeld M, et al. Indication-specific immunomodulatory effects of extracorporeal photopheresis: A pilot study in heart transplanted patients. Journal of clinical apheresis. 2018.

16. Dieterlen MT, Bittner HB, Pierzchalski A, et al. Immunological monitoring of extracorporeal photopheresis after heart transplantation. Clinical and experimental immunology. 2014;176:120-128. 
17. Denney HA, Whittle RJ, Lai J, et al. Regulatory t cells in chronic graft-versus-host disease after extracorporeal photopheresis: Correlation with skin and global organ responses, and ability to taper steroids. Transplantation. 2017;101:204-211.

18. Atta M, Papanicolaou N, Tsirigotis P. The role of extracorporeal photopheresis in the treatment of cutaneous t-cell lymphomas. Transfusion and apheresis science : official journal of the World Apheresis Association : official journal of the European Society for Haemapheresis. 2012;46:195-202.

19. Edelson R, Wu Y, Schneiderman J. American council on ecp (ace): Why now? Journal of clinical apheresis. 2018;33:464-468.

20. Berger C, Hoffmann K, Vasquez JG, et al. Rapid generation of maturationally synchronized human dendritic cells: Contribution to the clinical efficacy of extracorporeal photochemotherapy. Blood. 2010;116:4838-4847.

21. Gonzalez AL, Berger CL, Remington J, et al. Integrin-driven monocyte to dendritic cell conversion in modified extracorporeal photochemotherapy. Clinical and experimental immunology. 2014;175:449-457.

22. Durazzo TS, Tigelaar RE, Filler R, et al. Induction of monocyte-to-dendritic cell maturation by extracorporeal photochemotherapy: Initiation via direct platelet signaling. Transfusion and apheresis science : official journal of the World Apheresis Association : official journal of the European Society for Haemapheresis. 2014;50:370-378.

23. Ventura A, Vassall A, Robinson E, et al. Extracorporeal photochemotherapy drives monocyteto-dendritic cell maturation to induce anticancer immunity. Cancer research. 2018;78:4045-4058.

24. Futterleib JS, Feng H, Tigelaar RE, et al. Activation of gilz gene by photoactivated 8methoxypsoralen: Potential role of immunoregulatory dendritic cells in extracorporeal photochemotherapy. Transfusion and apheresis science : official journal of the World Apheresis Association : official journal of the European Society for Haemapheresis. 2014;50:379-387.

25. Hannani D, Gabert F, Laurin D, et al. Photochemotherapy induces the apoptosis of monocytes without impairing their function. Transplantation. 2010;89:492-499.

26. Morelli AE. The immune regulatory effect of apoptotic cells and exosomes on dendritic cells: Its impact on transplantation. American journal of transplantation : official journal of the American Society of Transplantation and the American Society of Transplant Surgeons. 2006;6:254-261.

27. Penberthy KK, Juncadella IJ, Ravichandran KS. Apoptosis and engulfment by bronchial epithelial cells. Implications for allergic airway inflammation. Annals of the American Thoracic Society. 2014;11 Suppl 5:S259-262.

28. PrabhuDas MR, Baldwin CL, Bollyky PL, et al. A consensus definitive classification of scavenger receptors and their roles in health and disease. J Immunol. 2017;198:3775-3789.

29. Prabhudas M, Bowdish D, Drickamer K, et al. Standardizing scavenger receptor nomenclature. J Immunol. 2014;192:1997-2006.

30. Kroemer G, Zitvogel L. Death, danger, and immunity: An infernal trio. Immunological reviews. 2007;220:5-7.

31. Obeid $M$, Tesniere A, Ghiringhelli $F$, et al. Calreticulin exposure dictates the immunogenicity of cancer cell death. Nature medicine. 2007;13:54-61.

32. Zitvogel L, Kepp O, Senovilla L, et al. Immunogenic tumor cell death for optimal anticancer therapy: The calreticulin exposure pathway. Clinical cancer research : an official journal of the American Association for Cancer Research. 2010;16:3100-3104.

33. Kepp O, Galluzzi L, Martins I, et al. Molecular determinants of immunogenic cell death elicited by anticancer chemotherapy. Cancer metastasis reviews. 2011;30:61-69.

34. Lui $G$, Manches $O$, Chaperot $L$, et al. Preparation of purified lymphoma cells suitable for therapy. Cytotherapy. 2004;6:235-243.

35. Suchin KR, Cassin M, Washko R, et al. Extracorporeal photochemotherapy does not suppress $\mathrm{t}$ - or b-cell responses to novel or recall antigens. Journal of the American Academy of Dermatology. 1999;41:980-986. 
36. Hannani D. Extracorporeal photopheresis: Tolerogenic or immunogenic cell death? Beyond current dogma. Frontiers in immunology. 2015;6:349.

37. Emerson RO, Mathew JM, Konieczna IM, et al. Defining the alloreactive $t$ cell repertoire using high-throughput sequencing of mixed lymphocyte reaction culture. PloS one. 2014;9:e111943.

38. Heinrich B, Klein J, Delic M, et al. Immunogenicity of oncolytic vaccinia viruses jx-gfp and tg6002 in a human melanoma in vitro model: Studying immunogenic cell death, dendritic cell maturation and interaction with cytotoxic t lymphocytes. OncoTargets and therapy. 2017;10:23892401.

39. Garg AD, Krysko DV, Vandenabeele P, et al. Hypericin-based photodynamic therapy induces surface exposure of damage-associated molecular patterns like hsp70 and calreticulin. Cancer immunology, immunotherapy : CII. 2012;61:215-221.

40. Garg AD, Krysko DV, Verfaillie T, et al. A novel pathway combining calreticulin exposure and atp secretion in immunogenic cancer cell death. The EMBO journal. 2012;31:1062-1079.

41. Wemeau M, Kepp O, Tesniere A, et al. Calreticulin exposure on malignant blasts predicts a cellular anticancer immune response in patients with acute myeloid leukemia. Cell death \& disease. 2010;1:e104.

42. Arosa FA, de Jesus $\mathrm{O}$, Porto $\mathrm{G}$, et al. Calreticulin is expressed on the cell surface of activated human peripheral blood $\mathrm{t}$ lymphocytes in association with major histocompatibility complex class $\mathrm{i}$ molecules. The Journal of biological chemistry. 1999;274:16917-16922.

43. Ghiringhelli F, Bruchard M, Chalmin F, et al. Production of adenosine by ectonucleotidases: A key factor in tumor immunoescape. Journal of biomedicine \& biotechnology. 2012;2012:473712.

44. Just $U$, Dimou E, Knobler R, et al. Leucocyte scintigraphy with 111in-oxine for assessment of cell trafficking after extracorporeal photopheresis. Experimental dermatology. 2012;21:443-447.

45. Lamioni A, Parisi F, Isacchi G, et al. The immunological effects of extracorporeal photopheresis unraveled: Induction of tolerogenic dendritic cells in vitro and regulatory t cells in vivo. Transplantation. 2005;79:846-850.

46. Kepp O, Senovilla L, Vitale I, et al. Consensus guidelines for the detection of immunogenic cell death. Oncoimmunology. 2014;3:e955691.

47. Summers deLuca L, Gommerman JL. Fine-tuning of dendritic cell biology by the tnf superfamily. Nature reviews Immunology. 2012;12:339-351.

48. Sallusto F, Lanzavecchia A. Efficient presentation of soluble antigen by cultured human dendritic cells is maintained by granulocyte/macrophage colony-stimulating factor plus interleukin 4 and downregulated by tumor necrosis factor alpha. The Journal of experimental medicine. 1994;179:1109-1118.

49. Boltjes A, van Wijk F. Human dendritic cell functional specialization in steady-state and inflammation. Frontiers in immunology. 2014;5:131. 


\section{Figure legends}

\section{Figure 1: PUVA-induced resting and activated T cell apoptosis}

The effect of PUVA treatment on the apoptosis of resting and alloreactive-activated T lymphocytes was measured by flow cytometry. A) Example of AnnexinV/7AAD analysis gated on CD3+ $T$ lymphocytes, for non-treated (NT) or PUVA-treated alloreactive activated T cells. In this setting, early apoptotic cells are AnnV+/7AADneg, late apoptotic cells are 7AAD+ cells. B) Mean and standard deviation of the percentages of dead cells (7AAD+ and/or AnnV+ cells) measured after 24 and $48 \mathrm{~h}$ hours in 22 (resting cells) and 16 (alloreactive-activated cells) independent experiments (healthy volunteers 'cells).

\section{Figure 2: Calreticulin expression and HMGB1 release induced by PUVA-treatment of T} cells

The surface expression of calreticulin was measured by flow cytometry, on resting and alloreactiveactivated T lymphocytes, after gating on 7AAD negative cells that retained their membrane integrity. A) Example of CRT expression on non-treated (NT, dark histogram) and PUVA-treated (light grey histogram) alloreactive activated T cells. B) Analysis of surface calreticulin expression on resting and activated T lymphocytes treated or not with PUVA and incubated for 24or 48 hours. C) HMGB1 was measured by ELISA in supernatants from cultures of resting and activated T lymphocytes treated or not with PUVA and incubated for 24 or 48 hours. In $B$ and D, data represent mean+/-SD of $n=29$ (CRT, 24h), $n=18$ (CRT, 48h), n=13 (HMGB1, 24h), n=24 (HMGB1, resting cells, 48h), and n=9 (HMGB1, activated cells, $48 \mathrm{~h}$ ) experiments. Statistical differences between the groups were analyzed by Annova.

Figure 3: Calreticulin expression upon PUVA treatment of PBMC from GVHD patients PBMC from patients with GVHD were treated or not with PUVA, and incubated for $24 \mathrm{~h}$, before measurement of calreticulin expression at the surface of 7AAD negative T cells. $A$ and $B$ ) calreticulin expression at the surface of activated (HLA-DR+) and non-activated (HLA-DRneg) T lymphocytes from GVHD patient PBMC. Light grey graphs represent NT sample, and dark graphs represent PUVA treated samples.

Figure 4: Phagocytosis by Macrophages and MoDC of PUVA-treated T cells; maturation of MoDC

Resting and activated T cells were PUVA-treated, incubated 24h, and then stained with CFSE before being mixed with APC for phagocytosis assay. After incubation, cells were harvested, and phagocytosis was determined as the percentage of CFSE positive cells among CD14 (macrophages) or CD209 (MoDC) positive CD3 negative cells (to exclude APC-T cell conjugates). A and B) representative experiment ( 1 hour incubation), and $C$ and $D$ ) mean of 5 and 6 independent experiments with macrophages $(A, C)$ and MoDC $(B, D)$, respectively. Significant differences between resting and activated PUVA-treated T cell phagocytosis are indicated by stars (Annova). MoDC maturation was quantified my measuring the mean fluorescence intensity of CD40 and CD80 after a 24-hour incubation in the same conditions. E) Fold increase of CD40 and CD80 mean fluorescent intensities normalized to the control condition (MoDC in medium). Bars represent mean+/-SD of 9 experiments. Statistical differences between the groups were analyzed by Annova. 
Figure 5: Proliferation and polarization of naive T cells induced by MoDC in contact with PUVA-treated T cells

Purified resting and activated T cells were PUVA-treated, incubated $24 \mathrm{~h}$, and mixed with autologous MoDC for $24 \mathrm{~h}$ before addition of allogeneic naive CD4+ T cells, as depicted in A. B) $3 \mathrm{H}$-Thymidine was added for $18 \mathrm{~h}$ at the end of 6 -day incubation, to measure cell proliferation. Negative control: MoDC preincubated with medium; positive control: MoDC preincubated with Polyl:C) C) T cell polarization was evaluated by measuring cytokines (cytometric bead array) secreted in culture supernatants after a restimulation with PMA and lonomycine. Bars represent mean+/-SD of 4 experiments (B) and 8 experiments $(C)$. Statistical differences between the groups were analyzed by Annova.

\section{Additional supporting information}

Additional supporting information are found in the supplementary figure file (pdf) 